\title{
Efficient Bistatic SAR Raw Signal Simulator of Extended Scenes
}

\author{
Liang Yang, ${ }^{1,2}$ Weidong Yu, ${ }^{1}$ Shichao Zheng, ${ }^{1,2}$ and Lei Zhang ${ }^{1,2}$ \\ ${ }^{1}$ Institute of Electronics, Chinese Academy of Sciences, Beijing 100190, China \\ ${ }^{2}$ University of the Chinese Academy of Sciences, Beijing 100039, China \\ Correspondence should be addressed to Liang Yang; yangliang_mail@163.com
}

Received 17 September 2013; Revised 24 December 2013; Accepted 24 December 2013; Published 10 February 2014

Academic Editor: Dau-Chyrh Chang

Copyright (C) 2014 Liang Yang et al. This is an open access article distributed under the Creative Commons Attribution License, which permits unrestricted use, distribution, and reproduction in any medium, provided the original work is properly cited.

\begin{abstract}
Bistatic SAR system is a new mode that allocates the radar transmitter and receiver on different platforms and has more advantages compared to the monostatic case. However, the existing bistatic SAR raw data simulator in the frequency domain can only handle the case of translation invariant system. In this paper, an efficient 2D frequency-domain raw data simulator of extended scenes for bistatic SAR of translation variant system is proposed by a geometric transformation method for the first time, where inverse STOLT interpolation is used to formulate the range migration terms. The presented simulator can accommodate the translation variant bistatic SAR system compared with existing bistatic SAR simulator. And it is more efficient than the time domain one by making use of Fast Fourier Transform (FFT). Simulation results for point targets and a real SAR image demonstrate its validity and effectiveness.
\end{abstract}

\section{Introduction}

Synthetic aperture radar (SAR) is a powerful remote sensing technique, which can work in all weather conditions and day and night [1]. Bistatic SAR is a SAR system that allocates the radar transmitter and receiver on different platforms [2-6]. It can obtain the reflectivity of the targets in all the directions with respect to the monostatic SAR system. In addition, the bistatic SAR has great potential for remote sensing applications in the fields of airborne and spaceborne remote sensing, such as high resolution imaging, wide-swath detecting, scene classification, and single pass crosstrack interferometry.

SAR raw data generator is essential for designing the new sensor, testing the imaging algorithm, and devising inversion algorithm, especially when real raw data are not available yet. Thus, the raw data simulator of extended scenes for the bistatic SAR becomes an increasingly wide interest in the remote sensing community. The SAR raw signal simulation methods mainly include time domain [7] and the two-dimensional (2D) frequency domain $[8,9]$. Time domain raw data simulation can be easily conceived, but the computational complexity cannot be tolerable, especially in the presence of extended scenes. Frequency domain method takes advantage of Fast Fourier Transform (FFT), and thus it highly reduces the computational load with respect to a time domain one. Due to the significance of the raw signal simulation, many efficient simulators for different imaging modes such as stripmap [8] and spotlight [9] in the 2D frequency domain have been proposed in recent years.

However, few studies are reported for the bistatic SAR raw data simulation. And most of them apply the time domain method to generate the raw data $[10,11]$. The efficient frequency domain simulation method is proposed in $[12,13]$ through the bistatic-to-monostatic formulas, nevertheless, the accuracy of the simulation is not high because of the hyperbolic approximation. Reference [14] also proposed a frequency domain bistatic SAR raw data simulator which is only available in the bistatic SAR translation invariant system. In this paper, an accurate and efficient raw data simulation for bistatic SAR of translation variant system is proposed in the $2 \mathrm{D}$ frequency domain based on the analytical bistatic point target reference spectrum (BPTRS) for the first time. The proposed approach can efficiently generate the raw data for the bistatic SAR translation variant system by a geometric transformation method, which highly reduces the computational load comparing to the time domain one by making use of FFT. 


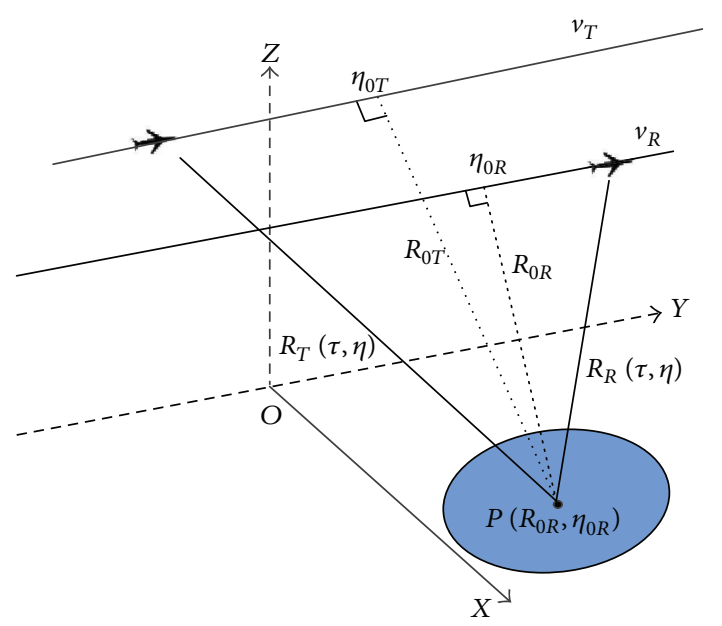

FIGURE 1: Bistatic SAR geometry.

The paper is organized as follows. In Section 2, the SAR bistatic point target reference spectrum (BPTRS) is derived. Section 3 shows the raw signal simulation procedure and theory of the bistatic SAR translation variant system. Section 4 exhibits plenty of simulation processing results to verify the presented method. And the computational complexity and phase error induced by the approximation in the simulation are also analyzed and compared in Section 5. Section 6 concludes this paper.

\section{Bistatic SAR Point Target Reference Spectrum}

A $2 \mathrm{D}$ signal spectrum is necessary to apply an efficient raw data simulation in the $2 \mathrm{D}$ frequency domain. In this section, we concentrate on the derivation of the SAR BPTRS using the principle of stationary phase (POSP). The bistatic SAR geometry is shown in Figure 1. The mathematical symbols and their definitions used in this paper are given as follows:

$\tau, \eta$ : variable of the range time domain and the Azimuth time domain,

$\left(R_{0 R}, \eta_{0 R}\right)$ : coordinate of the point target $P\left(R_{0 R}, \eta_{0 R}\right)$, given in receiver coordinate,

$\sigma\left(R_{0 R}, \eta_{0 R}\right)$ : reflectivity coefficients of the point target $P\left(R_{0 R}, \eta_{0 R}\right)$,

$\eta_{0 T}, \eta_{0 R}:$ zero Doppler time of the transmitter and receiver,

$R_{0 T}, R_{0 R}$ : closest slant ranges from transmitter and receiver to the point target,

$R_{T}(\tau, \eta), R_{R}(\tau, \eta)$ : slant ranges from transmitter and receiver to the point target,

$v_{T}, v_{R}$ : platform velocities of the transmitter and receiver,

$f_{\tau}, f_{\eta}$ : range and Azimuth frequency variables, c: signal transfer velocity, $f_{0}$ : carrier frequency of the transmitter signal,

$H_{0 T}, H_{0 R}$ : platform heights of the transmitter and receiver,

$K_{r}$ : chirp rate.

Suppose the received baseband signal from a point target $P\left(R_{0 R}, \eta_{0 R}\right)$ is expressed as

$$
\begin{aligned}
S(\tau, \eta)= & \sigma\left(R_{0 R}, \eta_{0 R}\right) \exp \left[j \pi K_{r}\left(\tau-\frac{R_{T}(\eta)+R_{R}(\eta)}{c}\right)^{2}\right] \\
& \times \exp \left[-j 2 \pi f_{0} \frac{R_{T}(\eta)+R_{R}(\eta)}{c}\right],
\end{aligned}
$$

where

$$
\begin{aligned}
& R_{T}(\eta)=\sqrt{R_{0 T}^{2}+\left(\eta-\eta_{0 T}\right)^{2} v_{T}^{2}} \\
& R_{R}(\eta)=\sqrt{R_{0 R}^{2}+\left(\eta-\eta_{0 R}\right)^{2} v_{R}^{2}}
\end{aligned}
$$

Performing Fourier Transform (FT) with respect to the range time and using the POSP, we have

$$
\begin{aligned}
S\left(f_{\tau}, \eta\right)= & \sigma\left(R_{0 R}, \eta_{0 R}\right) \exp \left[-j \frac{\pi f_{\tau}^{2}}{K_{r}}\right] \\
& \times \exp \left[-j 2 \pi\left(f_{0}+f_{\tau}\right) \frac{R_{T}(\eta)+R_{R}(\eta)}{c}\right] .
\end{aligned}
$$

To obtain the $2 \mathrm{D}$ spectrum, a further FT with respect to the Azimuth time is performed

$$
\begin{aligned}
S\left(f_{\tau}, f_{\eta}\right)= & \sigma\left(R_{0 R}, \eta_{0 R}\right) \exp \left[-j \frac{\pi f_{\tau}^{2}}{K_{r}}\right] \\
& \times \int \exp \left\{-j \phi_{B}\left(f_{\tau}, \eta\right)\right\} d \eta,
\end{aligned}
$$

where $\phi_{B}\left(f_{\tau}, \eta\right)$ is denoted by

$$
\phi_{B}\left(f_{\tau}, \eta\right)=2 \pi\left(f_{0}+f_{\tau}\right) \frac{R_{T}(\eta)+R_{R}(\eta)}{c}+2 \pi f_{\eta} \eta
$$

Then the stationary point of phase must be determined to evaluate the integral in (5) using the POSP. Equating the first derivative of (6) to zero gives

$$
\left.\frac{d \phi_{B}\left(f_{\tau}, \eta\right)}{d \eta}\right|_{\eta=\eta_{s}}=0
$$

Thus, the relationship between the Doppler frequency $f_{\eta}$ and the point of phase stationary $\eta_{s}$ can be expressed as

$$
f_{\eta}=-\frac{\left(f_{0}+f_{\tau}\right)}{c}\left[\frac{v_{T}^{2}\left(\eta_{s}-\eta_{0 T}\right)}{R_{T}\left(\eta_{s}\right)}+\frac{v_{R}^{2}\left(\eta_{s}-\eta_{0 R}\right)}{R_{R}\left(\eta_{s}\right)}\right] .
$$

It is difficult to directly evaluate the relation between the Doppler frequency $f_{\eta}$ and the point of phase stationary 
$\eta_{s}$ in (8) since (8) contains double square roots term. To circumvent the obstacle we separate $f_{\eta}$ into two components and each of them is related to $\eta_{s}$ by a single square root [3-6]. Based on the independent contribution of the transmitter and receiver, we can express the Doppler frequency as follows:

$$
f_{\eta}=f_{\eta T}+f_{\eta R}
$$

where

$$
\begin{aligned}
& f_{\eta T}=-\frac{\left(f_{0}+f_{\tau}\right)}{c} \frac{v_{T}^{2}\left(\eta_{s}-\eta_{0 T}\right)}{R_{T}\left(\eta_{s}\right)}, \\
& f_{\eta R}=-\frac{\left(f_{0}+f_{\tau}\right)}{c} \frac{v_{R}^{2}\left(\eta_{s}-\eta_{0 R}\right)}{R_{R}\left(\eta_{s}\right)} .
\end{aligned}
$$

Solving (10), we can obtain

$$
\begin{aligned}
& \eta_{s T}=\eta_{0 T}-\frac{c f_{\eta T} R_{0 T}}{\left(f_{0}+f_{\tau}\right) v_{T}^{2} \sqrt{1-\left(c^{2} f_{\eta T}^{2} / v_{T}^{2}\left(f_{0}+f_{\tau}\right)^{2}\right)}}, \\
& \eta_{s R}=\eta_{0 R}-\frac{c f_{\eta R} R_{0 R}}{\left(f_{0}+f_{\tau}\right) v_{R}^{2} \sqrt{1-\left(c^{2} f_{\eta R}^{2} / v_{R}^{2}\left(f_{0}+f_{\tau}\right)^{2}\right)}} .
\end{aligned}
$$

Substituting (11a) and (11b) into (2) and (3), respectively, the slant trajectories of the transmitter and receiver in the $2 \mathrm{D}$ frequency domain can be formulated as

$$
\begin{aligned}
& R_{T}\left(f_{\tau}, f_{\eta T}\right)=\frac{R_{0 T}\left(f_{0}+f_{\tau}\right) v_{T}}{\sqrt{\left(f_{0}+f_{\tau}\right)^{2}-c^{2} f_{\eta T}^{2}}}, \\
& R_{R}\left(f_{\tau}, f_{\eta R}\right)=\frac{R_{0 R}\left(f_{0}+f_{\tau}\right) v_{R}}{\sqrt{\left(f_{0}+f_{\tau}\right)^{2}-c^{2} f_{\eta R}^{2}}} .
\end{aligned}
$$

Substituting (12) into (8) yields

$$
f_{\eta}=\frac{\left(f_{0}+f_{\tau}\right)}{c}\left[\frac{v_{T}^{2}\left(\eta_{s}-\eta_{0 T}\right)}{R_{T}\left(f_{\tau}, f_{\eta T}\right)}+\frac{v_{R}^{2}\left(\eta_{s}-\eta_{0 R}\right)}{R_{R}\left(f_{\tau}, f_{\eta R}\right)}\right] .
$$

Then, we can achieve the analytical expression of the point of stationary phase by solving (13) for $\eta_{s}$

$$
\begin{aligned}
\eta_{s}= & \frac{c f_{\eta} R_{T}\left(f_{\tau}, f_{\eta T}\right) R_{R}\left(f_{\tau}, f_{\eta R}\right) /\left(f_{0}+f_{\tau}\right)}{R_{T}\left(f_{\tau}, f_{\eta T}\right) v_{R}^{2}+R_{R}\left(f_{\tau}, f_{\eta R}\right) v_{T}^{2}} \\
& +\frac{R_{T}\left(f_{\tau}, f_{\eta T}\right) v_{R}^{2} \eta_{0 R}+R_{R}\left(f_{\tau}, f_{\eta R}\right) v_{T}^{2} \eta_{0 T}}{R_{T}\left(f_{\tau}, f_{\eta T}\right) v_{R}^{2}+R_{R}\left(f_{\tau}, f_{\eta R}\right) v_{T}^{2}} .
\end{aligned}
$$

Substituting (14) into (10) for $\eta_{s}$, we can obtain the Doppler frequency of the transmitter and receiver contributed separately as follows:

$$
\begin{gathered}
f_{\eta T}=\frac{R_{0 R} v_{T}^{2}}{R_{0 T} v_{R}^{2}+R_{0 R} v_{T}^{2}} f_{\eta}+\frac{\left(f_{0}+f_{\tau}\right)}{c} \frac{v_{T}^{2} v_{R}^{2}\left(\eta_{0 R}-\eta_{0 T}\right)}{R_{0 T} v_{R}^{2}+R_{0 R} v_{T}^{2}}, \\
f_{\eta R}=\frac{R_{0 T} v_{R}^{2}}{R_{0 T} v_{R}^{2}+R_{0 R} v_{T}^{2}} f_{\eta}+\frac{\left(f_{0}+f_{\tau}\right)}{c} \frac{v_{T}^{2} v_{R}^{2}\left(\eta_{0 T}-\eta_{0 R}\right)}{R_{0 T} v_{R}^{2}+R_{0 R} v_{T}^{2}} .
\end{gathered}
$$

Finally, substituting (14) into (5), we can obtain the desired SAR BPTRS as follows:

$$
S\left(f_{\tau}, f_{\eta}\right)=\sigma\left(R_{0 R}, \eta_{0 R}\right) \exp \left\{-j \phi_{B}\left(f_{\tau}, f_{\eta}\right)\right\},
$$

where

$$
\begin{aligned}
\phi_{B}\left(f_{\tau}, f_{\eta}\right)= & \frac{2 \pi R_{0 T}}{c} \sqrt{\left(f_{0}+f_{\tau}\right)^{2}-\left(\frac{c f_{\eta T}}{v_{T}}\right)^{2}} \\
& +\frac{2 \pi R_{0 R}}{c} \sqrt{\left(f_{0}+f_{\tau}\right)^{2}-\left(\frac{c f_{\eta R}}{v_{R}}\right)^{2}} \\
& +2 \pi f_{\eta} \eta_{0 B}+\frac{\pi f_{\tau}^{2}}{K_{r}},
\end{aligned}
$$

where

$$
\eta_{0 B}=\frac{R_{0 T} v_{R}^{2} \eta_{0 R}+R_{0 R} v_{T}^{2} \eta_{0 T}}{R_{0 T} v_{R}^{2}+R_{0 R} v_{T}^{2}}
$$

when setting $R_{0 T}=R_{0 R}=R_{0}, v_{T}=v_{R}=v$, and $\eta_{0 T}=\eta_{0 R}=$ $\eta_{0}$, the bistatic SAR configuration changes into a monostatic case, and thus the phase of the BPTRS becomes

$$
\phi_{B}\left(f_{\tau}, f_{\eta}\right)=\frac{4 \pi R_{0}}{c} \sqrt{\left(f_{0}+f_{\tau}\right)^{2}-\left(\frac{c f_{\eta}}{2 v}\right)^{2}}+2 \pi f_{\eta} \eta_{0}+\frac{\pi f_{\tau}^{2}}{K_{r}} .
$$

It can be seen that the phase is the same as the monostatic SAR spectrum, which proves the accuracy of the SAR BPTRS. The first key to develop an efficient frequency domain bistatic SAR simulation approach is to achieve the $2 \mathrm{D}$ signal spectrum and the next key is how to generate the bistatic SAR raw data based on the $2 \mathrm{D}$ signal spectrum. We will introduce the raw data simulation procedure in detail in the next section.

\section{Bistatic SAR Raw Data Simulation Approach}

It is difficult to apply an efficient raw data simulation directly in the $2 \mathrm{D}$ frequency domain because the SAR BPTRS is dependent on the closest slant range from the point to the transmitter and receiver. Instead, the paper proposes a high efficient raw signal simulator based on the transformational bistatic SAR spectrum, which is reformulated by a geometric transformation method [6]. The geometric transformation map between transmitter and receiver is shown in Figure 2.

The closest slant range from the random point target in the scene to the receiver can be formulated as the sum of invariant term $R_{\text {ref_}_{-} R}$ and a linearly variant term $r$

$$
R_{0 R}=R_{\text {ref_R }}+r
$$

where $R_{\text {ref_R }}$ is the closest range from the scene center to the receiver and $r$ is the zero-offset receiver-to-target range variable. 


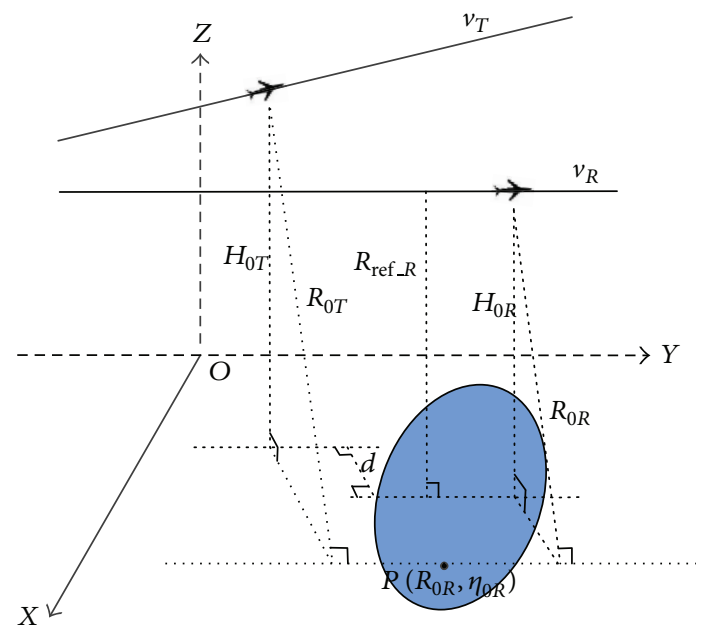

FIGURE 2: Geometric transformation map.

According to the geometric transformation map as shown in Figure 2, the closest slant range from target to the transmitter $R_{0 T}$ can be expressed as a function of $R_{0 R}$ and $r$ [6],

$$
\begin{aligned}
R_{0 T} & =\sqrt{\left(\sqrt{R_{0 R}^{2}-H_{0 R}^{2}}+d\right)^{2}+H_{0 T}^{2}} \\
& =\sqrt{\left(\sqrt{\left(R_{\text {ref } R}+r\right)^{2}-R_{0 R}^{2}}+d\right)^{2}+H_{0 T}^{2}},
\end{aligned}
$$

where $d$ denotes the projection of the constant offsets from the receiver to the transmitter in the range direction.

Expand (21) by using a first-order Taylor series in terms of $r / R_{\text {ref_R } R}$ and truncate them at the first-order term; (21) can be expressed as

$$
R_{0 T}=\alpha R_{\text {ref } \_}+\beta r
$$

where

$$
\begin{gathered}
\alpha=\sqrt{\left(\sqrt{1-\left(\frac{H_{0 R}}{R_{\text {ref_ } R}}\right)^{2}}+\frac{d}{R_{\text {ref } \_}}\right)^{2}+\left(\frac{H_{0 T}}{R_{\text {ref } \_}}\right)^{2}}, \\
\beta=\frac{R_{\text {ref } R}+\left(R_{\text {ref_R } R} / \sqrt{R_{\text {ref } R}^{2}-H_{0 R}^{2}}\right)}{\sqrt{\left(\sqrt{R_{\text {ref } \_}^{2}-H_{0 R}^{2}}+d\right)^{2}+H_{0 T}^{2}}} .
\end{gathered}
$$

Substituting (22) into (16), the bistatic SAR signal spectrum can be rewritten as

$$
S\left(f_{\tau}, f_{\eta}\right)=\sigma\left(R_{0 R}, \eta_{0 R}\right) \exp \left\{-j \phi_{B}\left(f_{\tau}, f_{\eta}\right)\right\},
$$

where

$$
\begin{aligned}
& \phi_{B}\left(f_{\tau}, f_{\eta}\right)=\frac{2 \pi\left(R_{0 R}-R_{\text {ref } \_R}\right)}{c} \\
& \times\left[\beta \sqrt{\left(f_{0}+f_{\tau}\right)^{2}-\left(\frac{c f_{\eta T}}{v_{T}}\right)^{2}}\right. \\
& \left.+\sqrt{\left(f_{0}+f_{\tau}\right)^{2}-\left(\frac{c f_{\eta R}}{v_{R}}\right)^{2}}\right] \\
& +\frac{2 \pi R_{\text {ref } R}}{c}\left[\alpha \sqrt{\left(f_{0}+f_{\tau}\right)^{2}-\left(\frac{c f_{\eta T}}{v_{T}}\right)^{2}}\right. \\
& \left.+\sqrt{\left(f_{0}+f_{\tau}\right)^{2}-\left(\frac{c f_{\eta R}}{v_{R}}\right)^{2}}\right] \\
& +2 \pi f_{\eta} \eta_{0 B}+\frac{\pi f_{\tau}^{2}}{K_{r}} .
\end{aligned}
$$

It can be seen from (25) that the square root terms are separated into two parts, one varies with the receiver-totarget shortest slant range, and the other one stands for the constant range and Azimuth coupling components.

Based on the signal spectrum model (24), we propose an efficient bistatic SAR raw data simulator; the procedure of simulation is shown in Figure 3.

The basic steps of the raw signal simulation are illustrated as follows.

(a) Projecting the reflectivity $\sigma\left(R_{0 R}, \eta_{0 R}\right)$ to the imaging plane, then the signal can be formulated as

$$
\begin{gathered}
g(\tau, \eta)=\iint G_{r}(\tau) \sigma\left(R_{0 R}, \eta_{0 R}\right) \delta\left(\tau-\tau_{0}\right) \\
\times \delta\left(\eta-\eta_{0 B}\right) d R_{0 R} d \eta_{0 R}
\end{gathered}
$$

where $G_{r}(\tau)$ is the antenna beam pattern in the range directions and $\delta(\tau)$ and $\delta(\eta)$ represent the sinc-like pulse envelope in the range and Azimuth directions, respectively. And $\tau_{0}$ denotes the delay from the receiver to the target corresponding to the scene center; that is,

$$
\tau_{0}=\frac{2\left(R_{0 T}-R_{\text {ref }-T}+R_{0 R}-R_{\text {ref } R}\right)}{c} .
$$




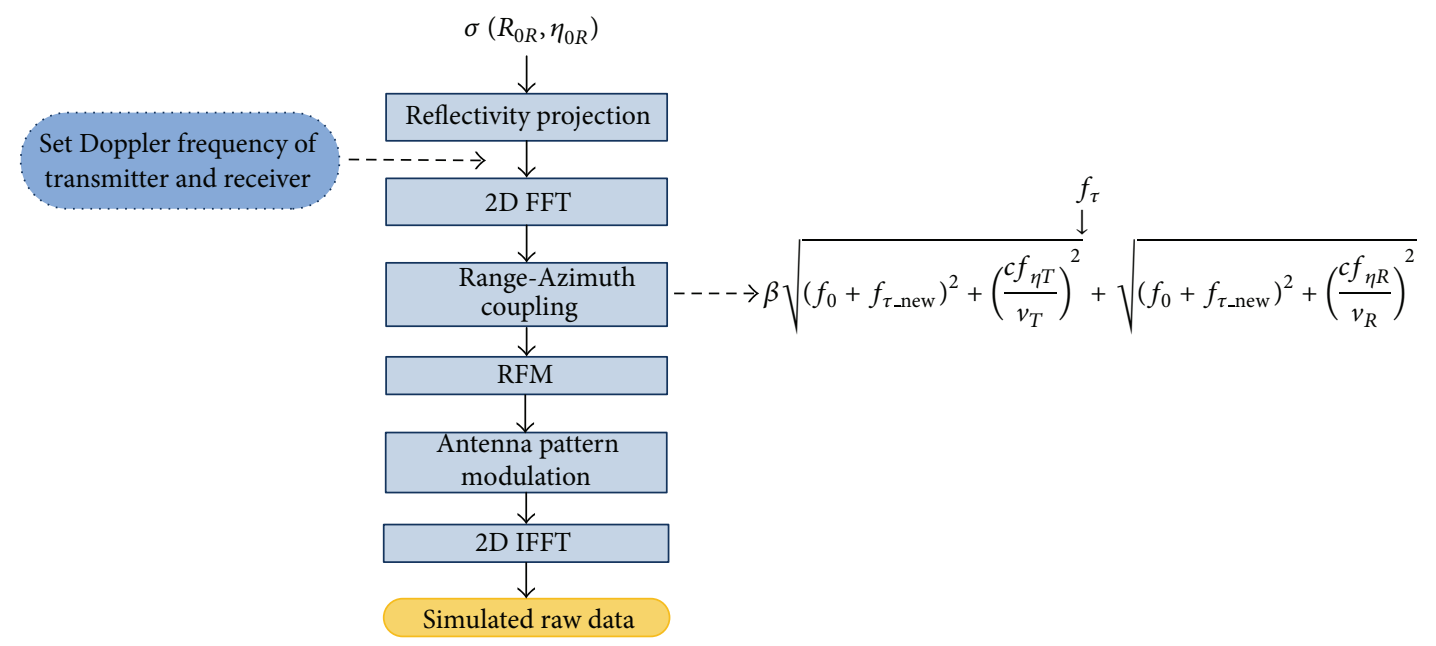

FIGURE 3: Flow of the proposed raw data simulator.

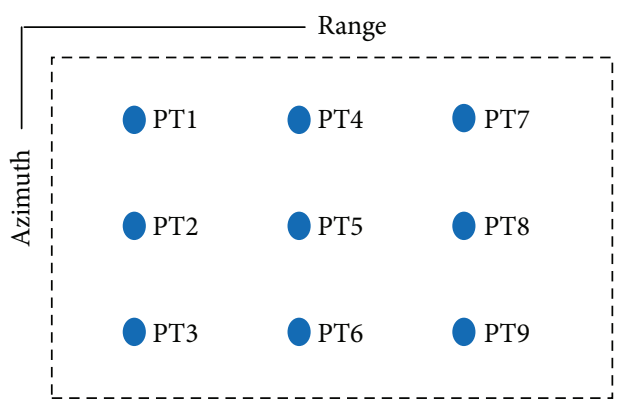

FIGURE 4: Simulation scene that contained nine point targets.

(b) Performing 2D FFT to transform the signal into the $2 \mathrm{D}$ frequency domain, thus the signal is given as

$$
\begin{aligned}
& G\left(f_{\tau}, f_{\eta}\right) \\
& =\iint G_{r}\left(\tau_{0}\right) \sigma\left(R_{0 R}, \eta_{0 R}\right) \\
& \quad \times \exp \left[-j 2 \pi\left(f_{0}+f_{\tau}\right) \tau_{0}-j 2 \pi f_{\eta} \eta_{0 B}\right] d R_{0 R} d \eta_{0 R} .
\end{aligned}
$$

(c) Carrying out the range frequency transition operation in the $2 \mathrm{D}$ frequency domain to simulate the rangeAzimuth coupling, that is,

$$
\begin{aligned}
& f_{\tau} \longrightarrow \beta \sqrt{\left(f_{0}+f_{\tau_{\text {nnew }}}\right)^{2}+\left(\frac{c f_{\eta T}}{v_{T}}\right)^{2}} \\
&+\sqrt{\left(f_{0}+f_{\tau_{\text {-new }}}\right)^{2}+\left(\frac{c f_{\eta R}}{v_{R}}\right)^{2}},
\end{aligned}
$$

we can achieve this step by inverse STOLT interpolation; however it can be seen from (29) that it is difficult to process directly owing to the double square root terms. Thus, we expand (29) using a Taylor series and keeping them to the first-order term. After this operation we can obtain

$$
\begin{gathered}
\beta \sqrt{\left(f_{0}+f_{\tau-\text { new }}\right)^{2}+\left(\frac{c f_{\eta T}}{v_{T}}\right)^{2}}+\sqrt{\left(f_{0}+f_{\tau_{- \text {new }}}\right)^{2}+\left(\frac{c f_{\eta R}}{v_{R}}\right)^{2}} \\
\approx \varphi_{0}\left(f_{\eta T}, f_{\eta R}\right) f_{0}+\varphi_{1}\left(f_{\eta T}, f_{\eta R}\right) f_{\tau_{\text {nnew }}},
\end{gathered}
$$

where

$$
\begin{gathered}
\varphi_{0}\left(f_{\eta T}, f_{\eta R}\right)=\left(\beta D_{T}+D_{R}\right), \\
\varphi_{1}\left(f_{\eta T}, f_{\eta R}\right)=\frac{\beta}{D_{T}}+\frac{1}{D_{R}},
\end{gathered}
$$

where

$$
\begin{aligned}
& D_{T}=\sqrt{1-\left(\frac{c f_{\eta T}}{V_{T} f_{0}}\right)^{2}}, \\
& D_{R}=\sqrt{1-\left(\frac{c f_{\eta R}}{V_{R} f_{0}}\right)^{2}} .
\end{aligned}
$$

Thus, we can get the mapping relation as follows:

$$
\frac{\left[1-\varphi_{0}\left(f_{\eta T}, f_{\eta R}\right)\right]+f_{\tau}}{\varphi_{1}\left(f_{\eta T}, f_{\eta R}\right)} \longrightarrow f_{\tau \text { new }} .
$$

Then, the mapping can be achieved in the frequency domain by the inverse STOLT interpolation. The signal after this operation is expressed as

$$
\begin{aligned}
G\left(f_{\tau \text { new }}, f_{\eta}\right)=\iint & G_{r}\left(\tau_{0}\right) \sigma\left(R_{0 R}, \eta_{0 R}\right) \\
& \times \exp \left[-j \phi_{B}\left(f_{\tau \text { new }}, f_{\eta}\right)\right] d R_{0 R} d \eta_{0 R},
\end{aligned}
$$


where

$$
\begin{aligned}
\phi_{B}\left(f_{\tau_{\_ \text {new }}}, f_{\eta}\right)= & \frac{2 \pi\left(R_{0 R}-R_{\text {ref_R }}\right)}{c} \\
& \times\left[\varphi_{0}\left(f_{\eta T}, f_{\eta R}\right) f_{0}+\varphi_{1}\left(f_{\eta T}, f_{\eta R}\right) f_{\tau_{\_ \text {new }}}\right] \\
& +2 \pi f_{\eta} \eta_{0 B} .
\end{aligned}
$$

The above processing procedures apply a Taylor series to get the convenient interpolation form, and the accuracy of this approximate formulation will be verified in Section 5.

(d) Performing Reference Function Multiplication (RFM) operation to simulate all the range-invariant phase, thus the function is given as

$$
\begin{gathered}
H_{\mathrm{RFM}}=\exp \left\{-\frac{2 \pi R_{\mathrm{ref}_{\_} R}}{c}\left[\alpha \sqrt{\left(f_{0}+f_{\tau}\right)^{2}-\left(\frac{c f_{\eta T}}{v_{T}}\right)^{2}}\right.\right. \\
\left.+\sqrt{\left(f_{0}+f_{\tau}\right)^{2}-\left(\frac{c f_{\eta R}}{v_{R}}\right)^{2}}\right] \\
\left.-\frac{\pi f_{\tau}^{2}}{K_{r}}\right\} .
\end{gathered}
$$

After the operation, the signal phase becomes

$$
\begin{aligned}
\phi_{B}\left(f_{\tau_{- \text {new }}}, f_{\eta}\right)= & \frac{2 \pi R_{\text {ref_R }}}{c} \\
& \times\left[\alpha \sqrt{\left(f_{0}+f_{\tau_{\text {_new }}}\right)^{2}-\left(\frac{c f_{\eta T}}{v_{T}}\right)^{2}}\right. \\
& \left.+\sqrt{\left(f_{0}+f_{\tau_{\text {_new }}}\right)^{2}-\left(\frac{c f_{\eta R}}{v_{R}}\right)^{2}}\right] \\
& \times\left[\varphi_{0}\left(f_{\eta T}, f_{\eta R}\right) f_{0}+\varphi_{1}\left(f_{\eta T}, f_{\eta R}\right) f_{\left.\tau_{- \text {ref } R}\right)}\right] \\
& +2 \pi f_{\eta} \eta_{0 B}+\frac{\pi f_{\tau_{- \text {new }}}^{2}}{K_{r}} .
\end{aligned}
$$

(e) To make sure that the generated raw data is precise, the skewness of the spectrum in the frequency domain must be simulated. Thus, the variance of the Azimuth beam pattern $G_{a}\left(f_{\eta}\right)$ with the range frequency $f_{\tau}$ should be considered. If neglecting the
TABLE 1: System parameters.

\begin{tabular}{lcc}
\hline & Transmitter & Receiver \\
\hline Carrier frequency & \multicolumn{2}{c}{$9.6 \mathrm{GHz}$} \\
Bandwidth & \multicolumn{2}{c}{$130 \mathrm{MHz}$} \\
Sample frequency & \multicolumn{2}{c}{$180 \mathrm{MHz}$} \\
PRF & \multicolumn{2}{c}{$600 \mathrm{~Hz}$} \\
Azimuth dimension of antenna & \multicolumn{2}{c}{$2 \mathrm{~m}$} \\
$\quad$ Platform velocity & $150 \mathrm{~m} / \mathrm{s}$ & $180 \mathrm{~m} / \mathrm{s}$ \\
Platform altitude & $8000 \mathrm{~m}$ & $7500 \mathrm{~m}$ \\
Depression angle & $53.13^{\circ}$ & $53.75^{\circ}$ \\
\hline
\end{tabular}

variation, it will result in the supporting area of the raw data deviating from the ideal area in the time domain. After the antenna pattern modulation, the signal is expressed as

$$
\begin{aligned}
\left(f_{\tau_{\text {_new }}}, f_{\eta}\right)=\iint & G_{r}\left(f_{\tau_{- \text {new }}}\right) \operatorname{rect}\left[\frac{f_{\tau_{\_ \text {new }}}}{B_{r}}\right] G_{a}\left(f_{\eta}\right) \\
& \times \sigma\left(R_{0 R}, \eta_{0 R}\right) \\
& \times \exp \left[-j \phi_{B}\left(f_{\tau_{- \text {new }}}, f_{\eta}\right)\right] d R_{0 R} d \eta_{0 R} .
\end{aligned}
$$

(f) Finally, the bistatic SAR raw signal of extended scenes can be generated by applying 2D Inverse Fast Fourier Transform (IFFT).

It can be seen that the above simulation procedures are based on inverse $\omega-\kappa$ algorithm, which can exactly simulate the range-Azimuth coupling. Thus, the proposed simulation method has high precision.

\section{Simulation Experiments}

To validate the accuracy of the simulated raw signal, a series of simulation experiment results is exhibited in this section. As we know, the most effective way to verify the precision of the generated raw data is to evaluate the corresponding imaging quality. First of all, raw data of simulation scene that contained nine point targets are simulated by both the proposed method and the time domain approach. The simulating parameters are given in Table 1, and the designed scene is shown in Figure 4. In Figure 4, the nine point targets are uniformly distributed on ground range plane, PT5 is located in the scene center, and with respect to the point target, PT2 and PT8 have the relative zero Doppler time: $-0.5 \mathrm{~s}$ and $0.5 \mathrm{~s}$, and PT4 and PT6 have the relative slant ranges $-190 \mathrm{~m}$ and $190 \mathrm{~m}$, respectively.

The focused result of the simulated raw data by the proposed method is shown in Figure 5(a), from which we can see that all the point targets are well focused at the expected position. The interpolated contours of PT9 are presented to evaluate the focus performance in detail, as shown in Figure 5(b). It can be seen that this point target is well compressed. Figures 5(c) and 5(d) compare the impulse response of PT9 in the same image with that achieved by the time domain method in the range and Azimuth directions, 
TABLE 2: Performance analysis of point targets.

\begin{tabular}{|c|c|c|c|c|c|c|}
\hline & \multicolumn{3}{|c|}{ Range } & \multicolumn{3}{|c|}{ Azimuth } \\
\hline & IRW (m) & $\operatorname{PSLR}(\mathrm{dB})$ & ISLR (dB) & IRW (m) & PSLR (dB) & ISLR $(\mathrm{dB})$ \\
\hline PT1 & 1.003 & -13.24 & -10.09 & 0.997 & -13.12 & -10.22 \\
\hline PT2 & 1.003 & -13.25 & -10.09 & 0.998 & -13.19 & -10.21 \\
\hline PT3 & 1.003 & -13.25 & -10.09 & 0.997 & -13.12 & -10.23 \\
\hline PT4 & 1.003 & -13.26 & -10.10 & 0.996 & -13.21 & -10.30 \\
\hline PT5 & 1.003 & -13.26 & -10.10 & 0.997 & -13.28 & -10.27 \\
\hline PT6 & 1.002 & -13.26 & -10.09 & 0.996 & -13.21 & -10.30 \\
\hline PT7 & 1.002 & -13.25 & -10.10 & 0.996 & -13.12 & -10.23 \\
\hline PT8 & 1.002 & -13.25 & -10.09 & 0.997 & -13.19 & -10.21 \\
\hline PT9 & 1.002 & -13.25 & -10.10 & 0.996 & -13.12 & -10.23 \\
\hline
\end{tabular}

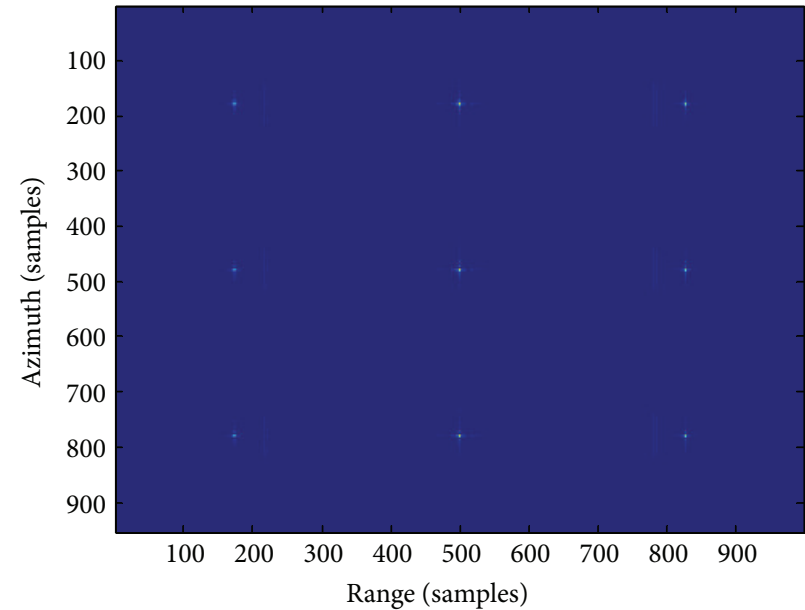

(a)

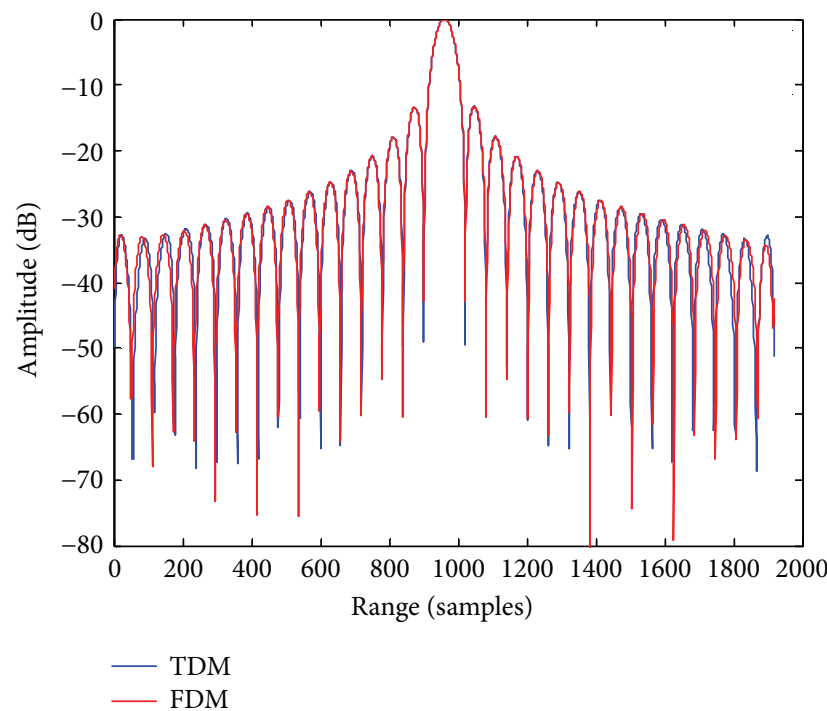

(c)

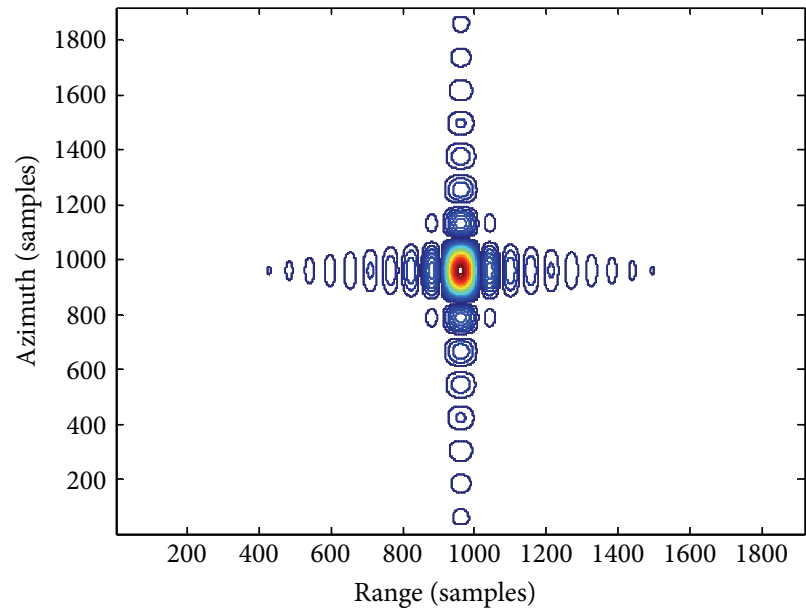

(b)

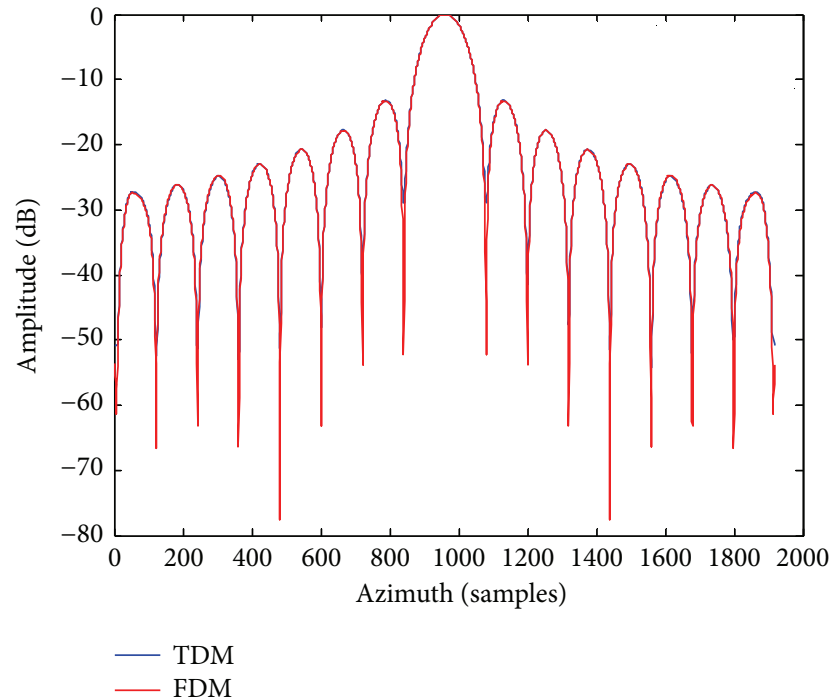

(d)

Figure 5: (a) The focused result of the generated raw data by the proposed simulator. (b) Interpolated contours of PT9. (c) Comparison with the range profiles of the resulting impulse response of PT9 of time domain method. (d) Comparison with the Azimuth profiles of the resulting impulse response of PT9 of time domain method. 


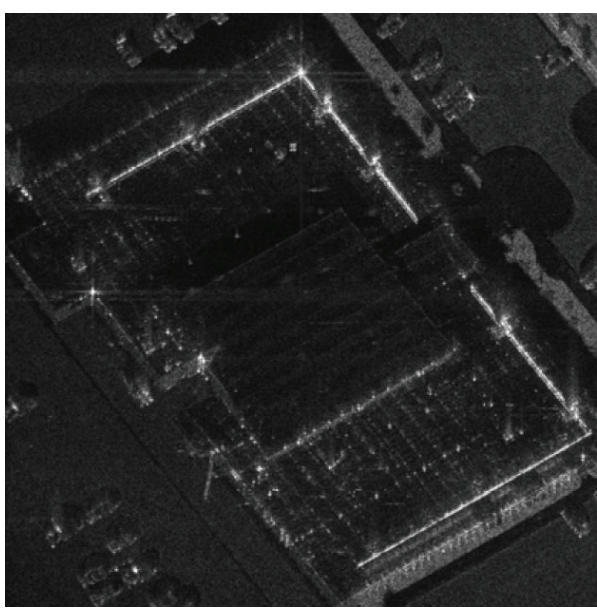

(a)

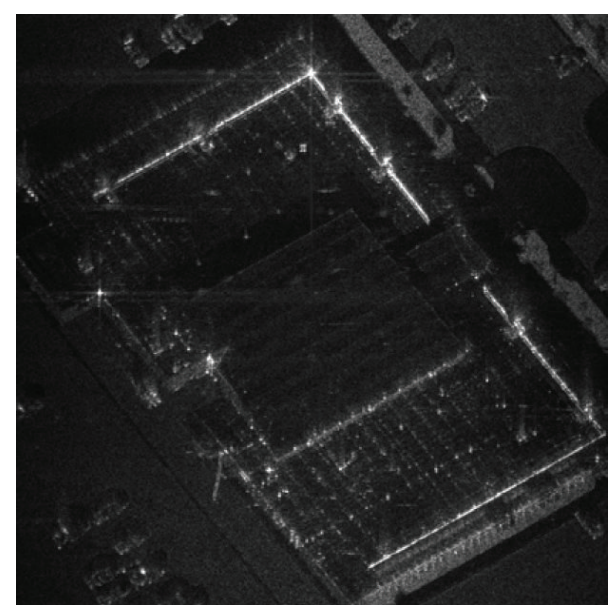

(b)

Figure 6: (a) The simulation scene imaging. (b) Focused result after processing the raw data generated by proposed frequency domain simulator.

TABle 3: Performance of point target (PT9) based on different simulation method.

\begin{tabular}{lcc}
\hline Quantitative norms & Time domain method & Proposed method \\
\hline IRW $(\mathrm{m})$ & & \\
Range & 1.001 & 1.002 \\
Azimuth & 0.999 & 0.996 \\
PSLR (dB) & & \\
Range & -13.27 & -13.25 \\
Azimuth & -13.15 & -13.12 \\
ISLR (dB) & & \\
Range & -10.08 & -10.10 \\
Azimuth & -10.28 & -10.23 \\
\hline
\end{tabular}

respectively. In Figures 5(c) and 5(d) we label the proposed method as FDM and denote the time domain approach as TDM. By contrast, we can observe that the two lines almost superposed each other, which proves the accuracy of the proposed method.

To quantify the focus performance more specifically, the analysis results that included the impulse response width (IRW), peak side-lobe ratio (PSLR), and integrated side-lobe ratio (ISLR) of all the point targets are listed in Table 2. Furthermore, the performance of PT9 is used as criterion to quantify the precision of processing with respect to the time domain method, as shown in Table 3. From it, we can see that the measured IRW in range and Azimuth are very close to the values of time domain simulator. The maximum deviation of PSLR in range and Azimuth is less than $0.03 \mathrm{~dB}$ with respect to the values of the time domain simulator. And the measured ISLR in range and Azimuth deviate from the time domain simulator values by no more than $0.05 \mathrm{~dB}$. The above results show that the measurements of target parameters agree nicely with the time domain method, which validates that the proposed frequency domain approach can accurately simulate the bistatic SAR raw signal.
Furthermore, a real extended scene simulation is carried out to verify the present simulation method. In the simulation, we used a real SAR image as backscattering coefficients, as presented in Figure 6(a). The raw data of the extended scene is generated based on the proposed simulation procedures as shown in Figure 3. And Figure 6(b) shows the SAR image after the raw signal was processed. It can be observed that the focused result of the scene is almost the same with the features of the real SAR image in Figure 6(a), without any geometry and defocusing. The simulation results prove the precision of the simulated raw data of extended scenes.

\section{Efficiency and Phase Error Analysis}

The primary advantage of the proposed raw data simulator is computational efficiency with respect to the time domain one. We apply the complex multiplication number to compare the efficiency of the two approaches. And the computation of the proposed simulator can be expressed as

$$
N_{f}=10 N_{a} N_{r} \log _{2}\left(N_{a} N_{r}\right)+6 N_{a} N_{r}+2\left(2 M_{\mathrm{ker}}-1\right) N_{a} N_{r},
$$

where $M_{\mathrm{ker}}$ is the length of interpolation kernel. $N_{a}$ and $N_{r}$ are the size of simulated scenes in the Azimuth and range directions, respectively. The computation of the time domain simulator is

$$
N_{t}=N_{a}^{2} N_{r}^{2}
$$

For example, a simulated image has 4096 pixels in the Azimuth and range directions, respectively, and the interpolation length of proposed method $M_{\mathrm{ker}}$ is 8 . Thus, we can get the complex multiplications of the time domain method and frequency domain method is different as high as $10^{5}$ level, which demonstrates that the proposed approach shows the computational efficiency. 


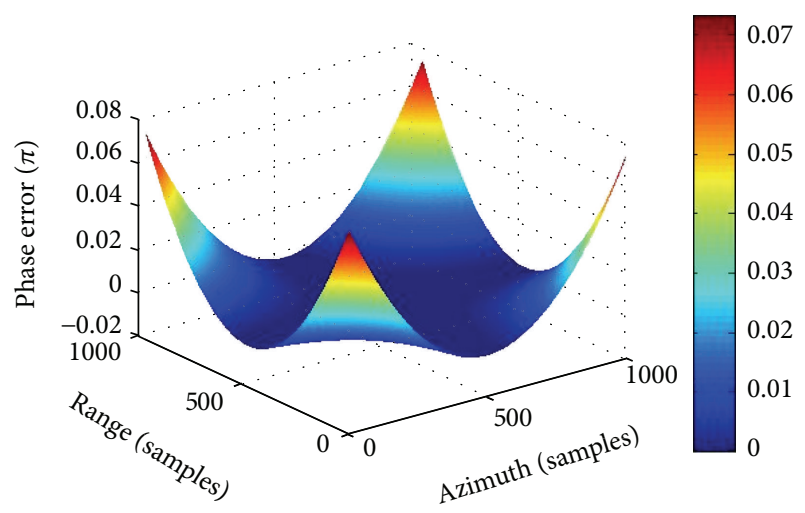

FIgURE 7: Phase error of the transformed spectrum.

In Section 3, we apply an approximate formulation (30) to use inverse STOLT interpolation conveniently. Thus, we need to calculate the phase error induced by the approximation to prove its accuracy and effectiveness. The accurate spectrum as shown in (24) is compared with approximate formulation as shown in (30), and we can obtain the phase error induced by the approximation as follows:

$$
\begin{aligned}
\varphi_{\text {error }}= & \frac{2 \pi \beta\left(R_{0 R}-R_{\text {ref } R}\right)}{c} \\
& \times\left\{\sqrt{\left(f_{0}+f_{\tau}\right)^{2}-\left(\frac{c f_{\eta T}}{v_{T}}\right)^{2}}-D_{T} f_{0}-\frac{1}{D_{T}} f_{\tau}\right\} \\
& +\frac{2 \pi\left(R_{0 R}-R_{\text {ref } R}\right)}{c} \\
& \times\left\{\sqrt{\left(f_{0}+f_{\tau}\right)^{2}-\left(\frac{c f_{\eta R}}{v_{R}}\right)^{2}}-D_{R} f_{0}-\frac{1}{D_{R}} f_{\tau}\right\} .
\end{aligned}
$$

The phase error is calculated according to the system parameters as shown in Table 1 , and the result is presented in Figure 7. It can be seen that the maximum phase error is about $0.07 \pi$, which is much smaller than the acceptable maximum phase error level which is $0.25 \pi$. The simulation results prove that the approximate spectrum is so precise that it can be used in our proposed bistatic SAR raw data simulator.

\section{Conclusion}

In this paper, an accurate and efficient bistatic SAR raw data simulator for extended scenes has been proposed. According to this approach, the raw data for the bistatic SAR translation variant system can be efficiently generated by a geometric transformation method. Therefore, the proposed approach can be applied in the real-time bistatic SAR raw signal simulator which can efficiently test the performance of the imaging processor.

\section{Conflict of Interests}

The authors declared that they have no conflict of interests regarding the publication of this paper.

\section{References}

[1] I. G. Cumming and F. H. Wong, Digital Processing of Synthetic Aperture Radar Data: Algorithms and Implementation, Artech House, Norwood, Mass, USA, 2005.

[2] G. Krieger and A. Moreira, "Spaceborne bi- and multistatic SAR: potential and challenges," IEE Proceedings: Radar, Sonar and Navigation, vol. 153, no. 3, pp. 184-198, 2006.

[3] H. Nies, O. Loffeld, and K. Natroshvili, "Analysis and focusing of bistatic airborne SAR data," IEEE Transactions on Geoscience and Remote Sensing, vol. 45, no. 11, pp. 3342-3349, 2007.

[4] R. Wang, O. Loffeld, Y. L. Neo, H. Nies, and Z. Dai, "Extending Loffeld's bistatic formula for the general bistatic SAR configuration," IET Radar, Sonar \& Navigation, vol. 4, no. 1, pp. 74-84, 2010.

[5] Y. L. Neo, F. Wong, and I. G. Cumming, "A two-dimensional spectrum for bistatic SAR processing using series reversion," IEEE Geoscience and Remote Sensing Letters, vol. 4, no. 1, pp. 93-96, 2007.

[6] R. Wang, O. Loffeld, H. Nies, S. Knedlik, and J. H. G. Ender, "Chirp-scaling algorithm for bistatic SAR data in the constantoffset configuration," IEEE Transactions on Geoscience and Remote Sensing, vol. 47, no. 3, pp. 952-964, 2009.

[7] A. Mori and F. De Vita, "A time-domain raw signal simulator for interferometric SAR," IEEE Transactions on Geoscience and Remote Sensing, vol. 42, no. 9, pp. 1811-1817, 2004.

[8] G. Franceschetti, M. Migliaccio, D. Riccio, and G. Schirinzi, "SARAS: a synthetic aperture radar (SAR) raw signal simulator," IEEE Transactions on Geoscience and Remote Sensing, vol. 30, no. 1, pp. 110-123, 1992.

[9] Y. Wang, Z. Zhang, and Y. Deng, "Squint spotlight SAR raw signal simulation in the frequency domain using optical principles," IEEE Transactions on Geoscience and Remote Sensing, vol. 46, no. 8, pp. 2208-2215, 2008.

[10] F. Comblet, F. Pellen, A. Baussard, and A. Khenchaf, "Bistatic SAR: theory and simulation," in Proceedings of the IEEE Antennas and Propagation Society International Symposium, vol. 2, pp. 664-667, July 2005.

[11] Y. Wu, H. Wang, and X. Jia, "Study and simulation on bistatic SAR," in Proceedings of the 7th International Symposium on Antennas, Propagation and EM Theory (ISAPE '06), pp. 1-4, Guilin, China, October 2006.

[12] X. Weijie and Z. Jianjiang, "A raw signal simulator for bistatic SAR," Chinese Journal of Aeronautics, vol. 22, no. 4, pp. 434-443, 2009.

[13] X. Weijie and Z. Jianjiang, "A raw signal simulator for the squint mode bistatic," in Proceedings of the 9th International Conference on Signal Processing (ICSP '08), pp. 2275-2278, Beijing, China, October 2008.

[14] X. Qiu, D. Hu, L. Zhou, and C. Ding, "A bistatic SAR raw data simulator based on inverse $\omega$ - $\kappa$ algorithm," IEEE Transactions on Geoscience and Remote Sensing, vol. 48, no. 3, pp. 1540-1547, 2010. 

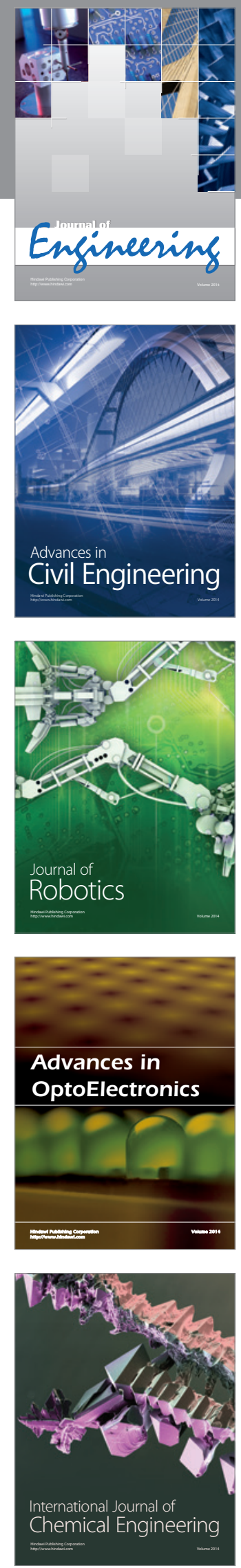

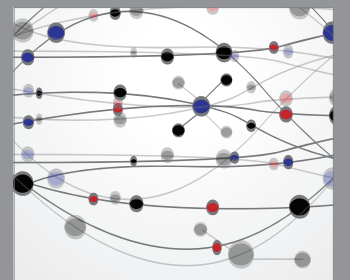

The Scientific World Journal
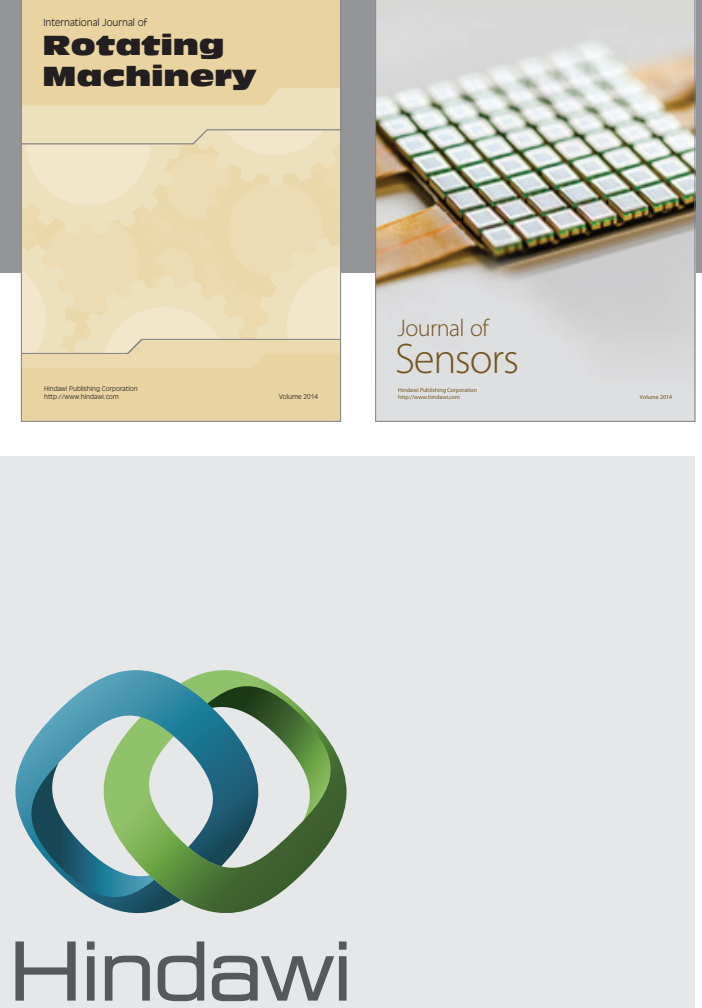

Submit your manuscripts at http://www.hindawi.com
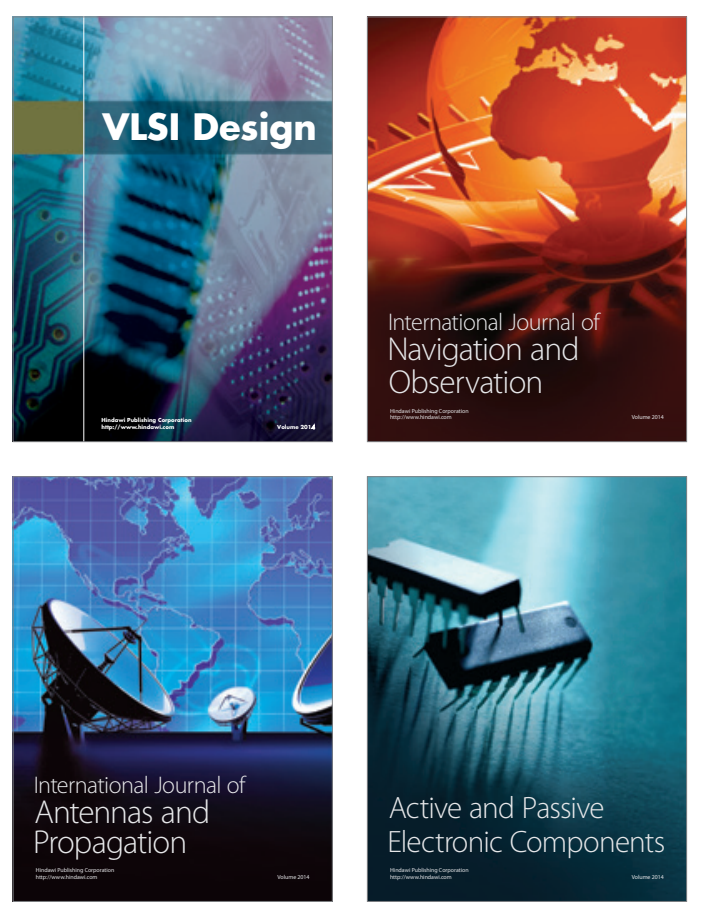
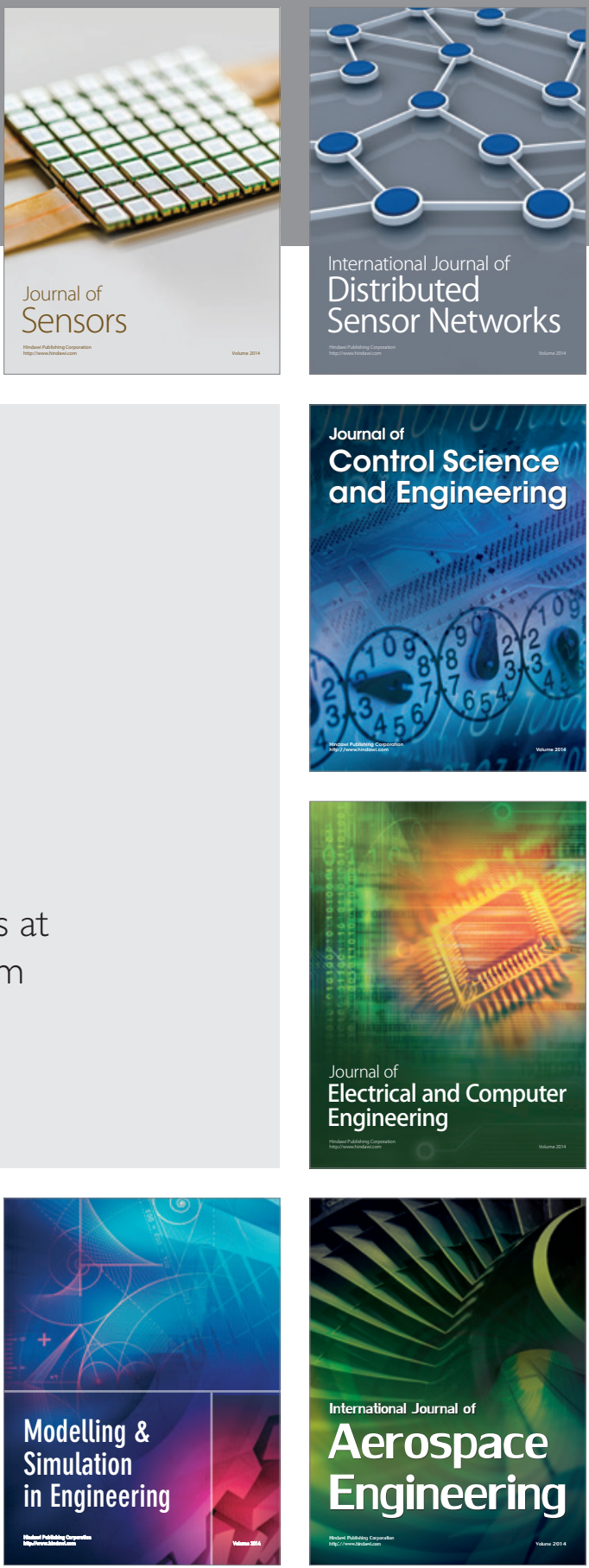

Journal of

Control Science

and Engineering
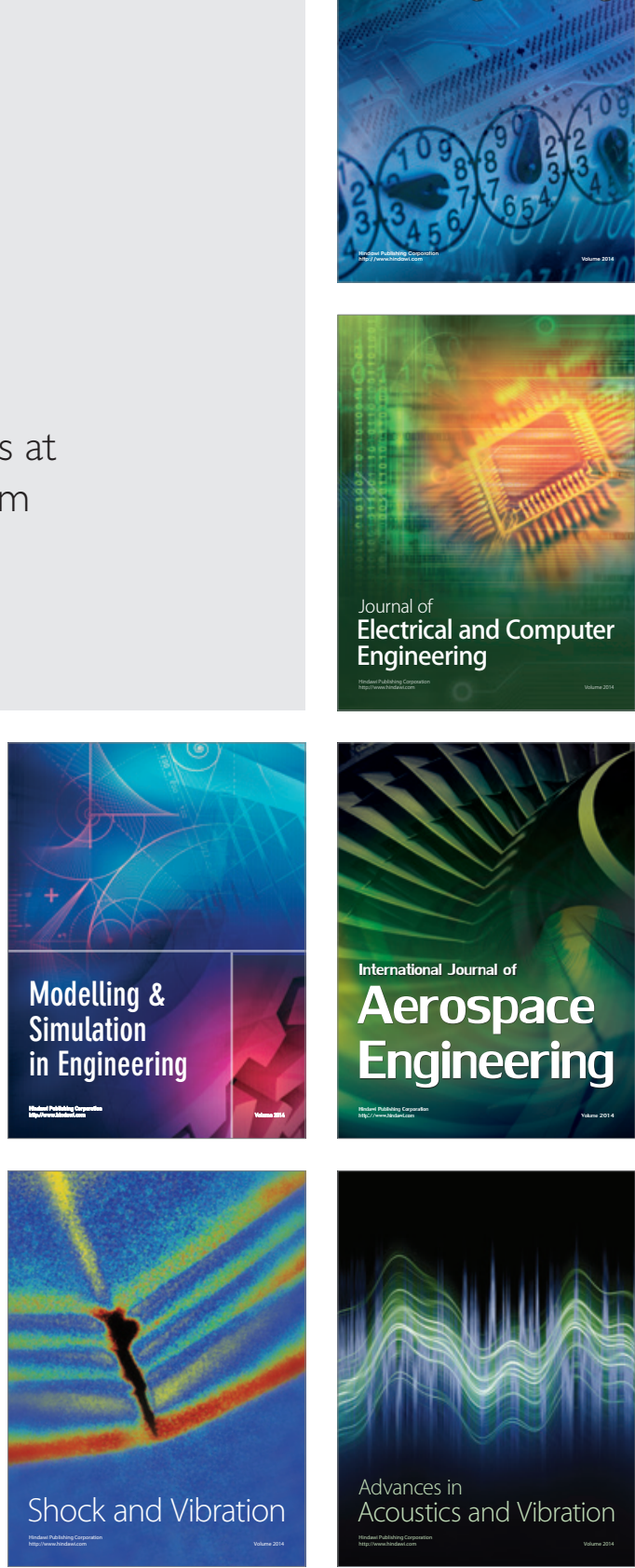